WALTER POHL

\title{
GESCHICHTE UND IDENTITÄT IM LANGOBARDENREICH
}

Das ca. 974 geschriebene Chronicon Salernitanum enthält eine Erzählung von der Bedeutung der langobardischen Vergangenheit für die süditalienischen Langobarden des 10. Jahrhunderts. Darin widerspricht ein Gastalde namens Rampho im Rat des Princeps Grimoald IV. (806-17) heftig dem Vorschlag, den heranrückenden Franken Tribut zu zahlen: „Viel besser ist es, kämpfend zu sterben, als hier unglücklicher zu leben. Habt Ihr, mein Fürst, nicht deutlich gelesen, wie unsere Vorväter ihre Wohnsitze verließen wegen des Tributs, den die Vandalen von ihnen verlangten?" ${ }^{1}$ Das ist ein deutlicher Hinweis auf die heute noch in einer Fassung der zweiten Hälfte des 7. Jahrhunderts erhaltene Origo gentis Langobardorum und die darin enthaltene langobardische Herkunftssage. ${ }^{2}$ Eine erweiterte Version dieses Herkunftsberichtes nahm vor $796 \mathrm{Pau}-$ lus Diaconus in seine Langobardengeschichte auf. ${ }^{3}$

Im Chronicon Salernitanum wird also vorausgesetzt, dass man in der langobardischen Führungsschicht des Fürstentums Benevent diese Sage kannte, ja sie sogar las, und daraus Argumente für politisches Handeln ableitete. Für uns verbinden sich in der Geschichte mehrere Zeitebenen: Um 974 wurde das Chronicon Salernitanum (vermutlich vom Abt des Benedikt-Klosters von Salerno) verfasst, während Amalfitaner und Capuaner um die Kontrolle der von Fraktionskämpfen erschütterten Stadt rangen; ${ }^{4}$ wenige Jahrzehnte später entstanden auch die beiden noch erhaltenen süditalienischen Handschriften, die den Text der Origo bieten. ${ }^{5}$ Um 810 spielt die Geschichte Ramphos im Widerstand der Langobarden von Benevent gegen die karolingische Expansion. Ebenfalls im Kontext des gespannten Verhältnisses zwischen Karl dem Großen und den

1 Chronicon Salernitanum 39 (ed. Ulla Westerbergh, Studia Latina Stockholmiensia 3, Stockholm/ Lund 1956) 41: Melius multo est pugnando mori quam hic infelicius vivere. Numquid non plane, mi princeps, legisti, quomodo propriis edibus patres nostri liquerunt propter vectigalia, que Guandalis ab eis exposcebant? Siehe dazu Walter Pohl, Werkstätte der Erinnerung. Montecassino und die langobardische Vergangenheit (MIÖG Erg. Bd. 39, Wien 2001) 170.

2 Origo gentis Langobardorum (ed. Georg Waitz, MGH SS rer. Lang., Hannover 1878) 1-6; oder: (ed. Annalisa Bracciotti, Biblioteca di cultura romanobarbarica 2, Rom 1998) 105-119. Siehe dazu Walter Pohl, Origo gentis (Langobarden), in: RGA 2. Aufl. 22 (2003) 183-189.

3 Paulus Diaconus, Historia Langobardorum I, 7-9 (ed. Georg Waitz, MGH SS rer. Lang., Hannover 1878) 12-187, hier 52f. Siehe Walter Pohl, Paulus Diaconus und die Historia Langobardorum: Text und Tradition, in: Historiographie im frühen Mittelalter, ed. Anton Scharer/Georg Scheibelreiter (Veröffentlichungen des Instituts für Ö̀sterreichische Geschichtsforschung 32, Wien 1994) 375-405; ders., Paolo Diacono e la costruzione dell'identità longobarda, in: Paolo Diacono - uno scrittore fra tradizione longobarda e rinnovamento carolingio, ed. Paolo Chiesa (Udine 2000) 413-426.

${ }_{4}$ Pohl, Werkstätte der Erinnerung 55-67; Huguette Taviani-Carozzi, La principauté Lombarde de Salerne, IX ${ }^{\mathrm{e}}-\mathrm{XI}^{\mathrm{e}}$ siècle 1 (Collection de l'École Française de Rome 152, Rome 1991) 62-95; vgl. auch Massimo Oldoni, Anonimo Salernitano del X secolo (Neapel 1972); Paolo Delogu, Mito di una città meridionale. Salerno, secoli VIII-XI (Napoli 1977).

${ }_{5}$ Es sind das die beiden Handschriften der Leges Langobardorum: Cava de' Tirreni, Biblioteca della Badia 4 (ca. 1005), sowie Madrid, Biblioteca Nacional 413 (sec. XI, 2. Viertel?): Pohl, Werkstätte der Erinnerung 108-151. 
Langobarden von Benevent hatte Paulus Diaconus die ridicula fabula von der langobardischen Namengebung nacherzählt; für die langobardische Identität im Süden Italiens blieb jene Zeit prägend, und Paulus wurde posthum zum Helden des Widerstands gegen die Karolinger stilisiert. ${ }^{6}$ Auch in der Zeit der Niederschrift der Origo in der zweiten Hälfte des 7. Jahrhunderts sind in der bayerischen Dynastie um Theodelinda, Gundeperga und Perctarit spezifische Interessen am Mythos zu entdecken. ${ }^{7}$ Die langobardische Herkunftssage selbst führt uns schließlich in eine mythische Vergangenheit, in der sich schattenhaft zwei weitere Zeitebenen abzeichnen. Die langobardische Königsliste geht zurück bis in die Zeit um 400, in die auch ein Vandalenkampf gut passen würde. Folgt man aber der Sage, so müssten sich die Namengebung der Langbärte durch Wodan und die Abwanderung aus Skandinavien zugetragen haben, bevor im 1. Jahrhundert unserer Zeitrechnung Langobarden erstmals an der unteren Elbe erwähnt sind.

Eine solche Zeittiefe ist bei der Frage nach ethnischen Identitäten des Mittelalters häufig; es geht um den Gebrauch der Ursprünge zur Sinnstiftung, Gemeinschaftsbindung und Herrschaftslegitimation. „Herrschaft braucht Herkunft“, hat Jan Assmann prägnant formuliert. ${ }^{8}$ Ein Jahrtausend langobardische Geschichte, das langobardische Identität begründet: mit dieser Beobachtung bewegen wir uns zunächst in einer durchaus etablierten Tradition von Forschungen über früh- und hochmittelalterliche Herkunftssagen und ihre identitätsstiftende Bedeutung. ${ }^{9}$ Dass ein kulturell vermitteltes subjektives Bekenntnis für ethnische Zugehörigkeit entscheidend ist, hat sich seit 1945 als Konsens in der Mittelalterforschung weitgehend durchgesetzt. ${ }^{10}$ Das erlaubt es erst, ethnische Prozesse als historische Abläufe zu verstehen. Die Forschungen der letzten Jahrzehnte über soziales Gedächtnis, Memoria und Erinnerungskultur haben geholfen, besser zu verstehen, wie Erinnerung Identität schafft, festigt oder verändert.11

Freilich, viele Fragen bleiben offen. Begriffe wie Tradition, Identität oder Erinnerung sind ja sehr unterschiedlich zu verstehen. Es fällt auf, dass das Problem der langobardischen Identität, wie viele Fragen der Langobardenforschung, in den letzten zwanzig Jahren sehr wenig diskutiert worden ist. ${ }^{2}$ Bei den Goten, aber auch bei Franken,

${ }^{6}$ Pohl, Werkstätte der Erinnerung 60-63 und 172; Huguette Taviani-Carozzi, Le souvenir et la légende de Paul Diacre, in: Haut Moyen Âge, Culture, Éducation et Société. Études offertes à Pierre Riché (Paris 1990) $555-573$.

7 Walter Pohl, La costituzione di una memoria storica: il caso dei Longobardi, in: Studi sulle società e le culture del medioevo per Gerolamo Arnaldi, ed. Ludovico Gatto/Paola Supino Martini (Florenz 2002) 563-580.

8 Jan Assmann, Das kulturelle Gedächtnis. Schrift, Erinnerung und politische Identität in den frühen Hochkulturen (München $\left.{ }^{2} 1997\right) 71$.

9 Siehe zuletzt Hans-Hubert Anton/Matthias Becher/Walter Pohl/Herwig Wolfram/Ian Wood, Origo gentis, in: RGA 22 (Berlin/New York 2003) 174-210. Vgl. auch Herwig Wolfram, Origo et Religio. Ethnic traditions and literature in early medieval texts, in: Early Medieval Europe 3 (1994) 19-38.

${ }_{10}$ Grundlegend: Reinhard Wenskus, Stammesbildung und Verfassung. Das Werden der frühmittelalterlichen gentes (Köln/Wien ${ }^{2} 1977$ ); Herwig Wolfram, Die Goten. Von den Anfängen bis zur Mitte des sechsten Jahrhunderts. Entwurf einer historischen Ethnographie (München ${ }^{4} 2000$ ); Patrick J. Geary, Europäische Völker im frühen Mittelalter. Zur Legende vom Werden der Nationen (Frankfurt 2001, engl.: The Myth of Nations: The Medieval Origins of Europe, Princeton 2001). Forschungsüberblick: Walter Pohl, Tradition, Ethnogenese und literarische Gestaltung: eine Zwischenbilanz, in: Ethnogenese und Überlieferung. Angewandte Methoden der Frühmittelalterforschung, ed. Karl Brunner/Brigitte Merta (Veröffentlichungen des Instituts für Österreichische Geschichtsforschung 31, Wien/München 1994) 9-26.

${ }^{11}$ Grundlegend für das frühe Mittelalter: Patrick J. Geary, Phantoms of Remembrance. Memory and Oblivion at the End of the First Millennium (Princeton 1994). Siehe auch James Fentress/Chris Wickham, Social Memory (Oxford 1992); Horst Wenzel, Hören und Sehen, Schrift und Bild: Kultur und Gedächtnis im Mittelalter (München 1995); Hochmittelalterliches Geschichtsbewußtsein im Spiegel nichthistoriographischer Quellen, ed. Hans-Werner Goetz (Berlin 1998).

12 Siehe den Beitrag von Jörg Jarnut in diesem Band; zu den Ausnahmen zählen Jörg Jarnut, Die langobardische Ethnogenese, in: Typen der Ethnogenese unter besonderer Berücksichtigung der Bayern 1, ed. 
Burgundern oder Alemannen war die Debatte viel lebhafter. ${ }^{13}$ Umstritten ist etwa, wie wandelbar ethnische Identitäten des Früh- und Hochmittelalters eigentlich waren. Breitenwirkung und Bindekraft des ethnischen Wir-Gefühls werden sehr unterschiedlich eingeschätzt; war ethnische Identität vielleicht nur rhetorisch beschworene ,ethnographische Ideologie', die den Zugang zu Standesprivilegien barbarischer Krieger sichern sollte?14 Haben wir überhaupt direkten Zugriff auf identitätswirksame Erinnerung, oder handelte es sich bei den erhaltenen Geschichtswerken, die vom Werden der Völker und Regna berichten, um hochtrabende literarische Entwürfe einiger weltfremder Gelehrter? ${ }^{15}$ Ein ähnlicher methodologischer Skeptizismus bei der Erforschung ethnischer Prozesse wird derzeit in der Archäologie debattiert: Lässt sich ethnische Identität überhaupt aus archäologischen Befunden ablesen? ${ }^{16}$ Zuweilen wird, in Reaktion darauf, auch wieder eine selbstverständliche und recht zeitresistente ethnische Zusammengehörigkeit der Barbarenvölker vorausgesetzt: War man doch einfach als Gote oder Langobarde geboren? Doch deutet alles darauf hin, dass ethnische Identität nicht die Voraussetzung, sondern das Resultat menschlichen Handelns und Denkens war. Identität ist immer der Differenz abgewonnen, sie entwirft soziale Grenzen, wo es in Wirklichkeit größere Vielfalt gab. ${ }^{17}$ Es geht darum, die Spuren dieses Handelns und der Kommunikation zu finden, durch die ethnische Zugehörigkeit für eine Gemeinschaft und ihre Umwelt Bedeutung erhielt. Solche Spuren finden sich sowohl in Texten als auch in archäologischen Befunden.

Bei den Langobarden entstand Identität aus einer doppelten Differenz: Einerseits war das die Differenz zwischen den Barbaren und ihrer römischen Umwelt, die in der langobardischen Identität lange Zeit quasi symbolisch konserviert wurde. Im langobardischen Süditalien des 9. und 10. Jahrhunderts erreichte die rhetorische Betonung lan-

Herwig Wolfram/Walter Pohl (Denkschriften der Österreichischen Akademie der Wissenschaften 201, Wien 1990) 97-103; ders., Die Landnahme der Langobarden in Italien aus historischer Sicht, in: Ausgewählte Probleme europäischer Landnahmen des Früh- und Hochmittelalters: methodische Grundlagendiskussion im Grenzbereich zwischen Archäologie und Geschichte 1, ed. Michael Müller-Wille/Reinhard Schneider (Vorträge und Forschungen 41, Sigmaringen 1993) 173-194; sowie Stefano Gasparri, Prima delle nazioni. Popoli, etnie e regni fra antichità e medioevo (Rom 1997) 141-160.

${ }_{13}$ Siehe z. B. für die Goten: Wolfram, Goten; Peter J. Heather, The Goths (Oxford/Cambridge-Mass. 1996); Patrick Amory, People and Identity in Ostrogothic Italy, 489-544 (Cambridge 1997); für die Burgunder: Ian N. Wood, Ethnicity and the ethnogenesis of the Burgundians, in: Typen der Ethnogenese unter besonderer Berücksichtigung der Bayern 1, ed. Herwig Wolfram/Walter Pohl (Denkschriften der Österreichischen Akademie der Wissenschaften 201, Wien 1990) 53-69; Patrick Amory, Names, ethnic identity, and community in fifth- and sixth-century Burgundy, in: Viator 25 (1994) 1-30; für die Franken: Ian N. Wood, Defining the Franks: Frankish origins in early medieval historiography, in: Concepts of National Identity in the Middle Ages, ed. Simon Forde/Lesley Johnson/Alan V. Murray (Leeds 1995) 47-57; Walter Pohl, Zur Bedeutung ethnischer Unterscheidungen in der frühen Karolingerzeit, in: Studien zur Sachsenforschung 12, ed. Hans-Jürgen Hässler (Oldenbourg 1999) 193-208; Alemannen: Helmut Castritius, Semnonen - Juthungen - Alemannen. Neues und (Altes) zur Herkunft und Ethnogenese der Alemannen, in: Die Franken und die Alemannen bis zur „Schlacht bei Zülpich“ (496/97), ed. Dieter Geuenich (RGA Erg. Bd. 19, Berlin/New York 1998) 349_ 366; Dieter Geuenich, Geschichte der Alemannen (Stuttgart/Berlin/Köln 1997); Überblick: Walter Pohl, Die Völkerwanderung. Eroberung und Integration (Stuttgart/Berlin/Köln 2002).

14 Amory, People and Identity.

15 Walter Goffart, The Narrators of Barbarian History, AD 550-880. Jordanes, Gregory of Tours, Bede and Paul the Deacon (Princeton 1988); On Barbarian Identity - Critical Approaches to Ethnogenesis Theory, ed. Andrew Gillett (Turnhout 2002).

${ }_{16}$ Sebastian Brather, Ethnische Identitäten als Konstrukte der frühgeschichtlichen Archäologie, in: Germania 78 (2000) 139-177. Siehe dazu den Beitrag von Volker Bierbrauer in diesem Band.

${ }_{17}$ Walter Pohl, Aux origines d'une Europe ethnique: Identités en transformation entre antiquité et moyen âge, in: Annales: Histoire, Sciences Sociales 60,1 (2005) 183-208; ders., Identität und Widerspruch. Gedanken zu einer Sinngeschichte des Frühmittelalters, in: Die Suche nach den Ursprüngen. Von der Bedeutung des frühen Mittelalters, ed. Walter Pohl (Forschungen zur Geschichte des Mittelalters 8, Wien 2004) 23-36. 
gobardischer Identität ihren Höhepunkt; hier waren aber auch die tatsächlichen Unterschiede zu den ,Romanen' am geringsten. Die moderne Geschichtsforschung hat bis vor kurzem das Anderssein der Langobarden als ausschlaggebendes Identitätsmerkmal betrachtet und demgegenüber eine zweite Differenz vernachlässigt, nämlich diejenige, die sowohl synchron als auch diachron zwischen den Langobarden herrschte. ${ }^{18}$ Eine solche simplifizierende Betrachtungsweise ist besonders bei der Untersuchung des Wandels der ,langobardischen Welt' in Italien zwischen 568 und 774 und darüber hinaus hinderlich.

Vor allem die pauschale Definition der Langobarden als Germanen hat lange ein irreführendes Interpretationsschema vorgegeben. ${ }^{19}$ Wenn all das, was an der langobardischen Gesellschaft und Kultur anders und nicht-klassisch ist, als germanisch verstanden wird, dann würde das letztlich bedeuten, dass es aus dem gemein-germanischen Erbe der Zeit vor 568 stammen muss. Was zum Beispiel König Rothari 643 in seinem Edikt an Rechtssatzungen aufzeichnen ließ, wäre daher im Wesentlichen voritalisches Gewohnheitsrecht aus jener Zeit, als die Langobarden noch im germanischen Siedlungsverband lebten. ${ }^{20}$ Arimannen werden erstmals in einem Gesetz aus dem elften Jahr Liutprands (723) erwähnt, aber da die Funktion eine germanische Bezeichnung trägt, müsste sie auf graue Vorzeit zurückgehen. ${ }^{21}$ In den Debatten der sechziger und siebziger Jahre hat sich die Forschung weitgehend von dem statischen Bild gelöst, das sich aus diesem Paradigma fast notwendigerweise ergibt. ${ }^{22}$ Dazu hat das Modell der Akkulturation gute Dienste geleistet. ${ }^{23}$ Doch impliziert das wiederum, dass das dynamische Element in Langobardenreich das spätrömische war, während das germanische im Lauf der Zeit zurückgedrängt wurde. Manche Phänomene sind auf diese Weise recht einleuchtend zu erklären, etwa das allmähliche Verschwinden der langobardischen Grabsitten im Lauf des 7. Jahrhunderts. Doch genügt wirklich ein solches bipolares Modell, das kulturellen und gesellschaftlichen Wandel im Langobardenreich im Wesentlichen aus der Interaktion zwischen Germanentum und Romanitas erklärt?

Zunächst ist es wichtig festzuhalten, dass die Langobarden in Italien von den Zeitgenossen nicht als Germanen angesehen wurden; schon gar nicht ist eine germanische Selbstzuordnung bezeugt. ${ }^{24}$ Paulus Diaconus erwähnt zwar, dass sich der Ursprung der Winniler, der späteren Langobarden, von den Völkern der Germanen herleitete, doch war das eine Sache der entfernten Vergangenheit. ${ }^{25}$ Der Germanenbegriff im kaiserzeit-

18 Ausführlicher dazu Walter Pohl, Le identità etniche nei ducati di Spoleto e Benevento, in: I Longobardi dei ducati di Spoleto e Benevento (Atti del XVI congresso internazionale di studi sull'alto medioevo, Spoleto 2003) 79-103.

19 Dazu und zum folgenden Walter Pohl, Vom Nutzen des Germanenbegriffes zwischen Antike und Mittelalter: eine forschungsgeschichtliche Perspektive, in: Akkulturation. Probleme einer germanisch-romanischen Kultursynthese in Spätantike und frühem Mittelalter, ed. Dieter Hägermann/Wolfgang Haubrichs/ Jörg Jarnut, unter Mitarbeit von Claudia Giefers (RGA Erg. Bd. 41, Berlin/New York 2004) 18-34.

${ }^{20}$ Forschungsüberblick: Gerhard Dilcher, Langobardisches Recht, in: Handwörterbuch zur Deutschen Rechtsgeschichte 2, ed. Adalbert Erler/Ekkehard Kaufmann (Berlin 1978) 1607-1618; Walter Pohl, Leges Langobardorum, in: RGA 2. Aufl. 18 (2001) 208-213.

${ }^{21}$ Liutprandi Leges 44 (ed. Friedrich Bluhme, MGH LL 4, Hannover 1868) 126f. Vgl. Jörg Jarnut, Beobachtungen zu den langobardischen arimanni und exercitales, in: Zeitschrift der Savigny-Stiftung für Rechtsgeschichte, Germanistische Abteilung 88 (1971) 1-28.

${ }_{22}$ Siehe dazu die Beiträge von Jörg Jarnut und Paolo Delogu in diesem Band.

${ }_{23}$ Zur Problematik der Akkulturation siehe künftig: Akkulturation. Probleme einer germanisch-romanischen Kultursynthese in Spätantike und frühem Mittelalter, ed. Dieter Hägermann/Wolfgang Haubrichs/ Jörg Jarnut, unter Mitarbeit von Claudia Giefers (RGA Erg. Bd. 41, Berlin/New York 2004).

${ }_{24}$ Walter Pohl, Der Germanenbegriff vom 3. bis 8. Jahrhundert - Identifikationen und Abgrenzungen, in: Zur Geschichte der Gleichung ,germanisch - deutsch', ed. Heinrich Beck/Dieter Geuenich/Heiko Steuer/ Dietrich Hakelberg (RGA Erg. Bd. 34, Berlin/New York 2004) 163-183.

${ }_{25}$ Paulus Diaconus, Historia Langobardorum I, 1, ed. Waitz 48: a Germanorum populis originem ducens. 
lichen Sinn war seit der Spätantike verschwunden und wurde allenfalls auf die Franken eingeschränkt gebraucht. Das ist nicht nur eine Frage der Begriffsgeschichte. Offenbar bestand kein Bedarf dafür, die nachrömischen Regna, ihren Reichsaufbau, ihr Recht oder ihre Kultur mit Verweis auf deren gemeinsame außerrömische Herkunft zu charakterisieren. Menschen germanischer Herkunft auf Reichsboden hatten im 6. oder 7. Jahrhundert mit ihren Vorfahren in den Wäldern Germaniens eben offenbar weniger gemeinsam als mit ihren Zeitgenossen romanischer Abstammung, und auch untereinander unterschieden sie sich wohl beträchtlich.

Entkleidet man nun den Germanenbegriff seiner irreführenden und immer noch ideologisch hochaufgeladenen Evidenz, wird deutlich, dass in der modernen Forschung das Attribut ,germanisch' im Langobardenreich sehr unterschiedliche Erscheinungen beschreiben kann: ${ }^{26}$ Erstens gehören dazu Dinge, deren Herkunft aus der kaiserzeitlichen Germania nachgewiesen oder zumindest angenommen werden kann, die Sprache etwa, aber auch manches an Tracht und Brauchtum, wobei bereits der jahrhundertelange Kulturaustausch zwischen Rom und der Germania zu berücksichtigen ist. Zweitens muss nicht alles, was außerrömischer Herkunft war, germanischen Ursprungs sein, sondern kann etwa aus der Steppenzone stammen, wie zum Beispiel die Technik des Reiterkampfes. Drittens mag manches Nichtklassische dennoch innerrömischer Herkunft sein, wie es bei Elementen des Vulgarrechtes oder bei für barbarischen Geschmack produziertem Kunsthandwerk angenommen wurde. Viertens greift selbst bei den Ausdrucksformen der ,barbarischen“ Kultur des 6. Jahrhunderts, also dem ,germanischen' Tierstil, den Goldbrakteaten oder den Runeninschriften, das Etikett ,germanisch" vielleicht zu kurz, weil es sich um eine internationale Kriegerkultur handelte, die eben nicht bloß Ausdruck germanischer Identität war (auch im Awarenreich blühte zum Beispiel gegen 600 der Tierstil). ${ }^{27}$ Fünftens aber, und das ist der wesentliche Einwand: Das Königreich der Langobarden in Italien war ebenso wie das der Franken oder der Westgoten (wenn auch vielleicht zunächst in geringerem Maß) eine Gesellschaft im Übergang, die in einer gewandelten Welt neue politische und kulturelle Lösungen finden musste. Die Ämter und Titel (etwa Duces und Gastalden), die Leges, die Dokumentation von Besitztransfers auf Pergamenturkunden, die Rolle der Kirche und der Klöster, die Bestattung vornehmer Laien innerhalb der Kirchen, die Goldblattkreuze, all das sind dynamische Elemente im Langobardenreich. Die Forschungsfrage nach ihrem ,germanischen“ oder ,romanischen“ Ursprung ist, wenn überhaupt, erst in zweiter Linie interessant. Wichtiger ist zunächst, ihre Entwicklung und Rolle im frühmittelalterlichen Kontext zu untersuchen und sich dabei den Blick für gesellschaftliche Innovation nicht durch die Frage nach den Ursprüngen zu verstellen.

Leitvorstellung bei der Darstellung der Geschichte der Langobarden sollte daher nicht ihr letztlich unveränderliches ,Wesen', ihre dem Wandel entrückte Identität sein. Im Gegenteil, die langobardische Identität wird erst dadurch als historische Leistung sichtbar, dass Brüche und Veränderungen in die Betrachtung einbezogen werden. Blicken wir nochmals mit dem Chronisten von Salerno zurück in die langobardische Vergangenheit. Das ist eine relativ gut rekonstruierbare Perspektive; wenige Jahrzehnte später wurden in Süditalien zwei der drei Leges-Handschriften angelegt, in denen bis heute die Origo gentis Langobardorum überliefert ist. ${ }^{28}$ Der Zeittiefe des Mythos entspricht tatsächlich eine Geschichte der Langobarden, die in der Zeit des Strabo und des Tacitus begann. ${ }^{29}$ In-

${ }_{26}$ Pohl, Zur Entwicklung.

27 Siehe den Beitrag von Ursula Heinrich-Tamaska in diesem Band.

${ }_{28}$ Siehe oben Anm. 5.

${ }_{29}$ Velleius Paterculus 2, 106 (ed. William S. Watt, Bibliotheca scriptorum Graecorum et Romanorum Teubneriana, Stuttgart/Leipzig ${ }^{2}$ 1998) 73; Strabo 7, 1, 3 (ed. Raoul Baladié, Paris 1989) 68; Tacitus, Germania 
sofern sind die Langobarden des Mythos keine literarische Fiktion. Freilich ist diese Geschichte mehrfach gebrochen. Das betrifft zunächst die auffallend lange Lücke von etwa 400 Jahren, die zwischen dem Langobardeneinfall von 166/67 und der nächsten Erwähnung bei Prokop und Jordanes in der Mitte des 6. Jahrhunderts liegt: Sind die Langobarden in Rugiland und Pannonien wirklich dasselbe Volk, das in der frühen Kaiserzeit an der unteren Elbe wohnte? ${ }^{30}$ Jüngst ist eine archäologische Diskussion über Kontinuität und Bruch in den Siedlungsverhältnissen in Südmähren und im nördlichen Niederösterreich entstanden, als um 500 die Langobarden sich hier durchsetzten. ${ }^{31}$ Vielleicht haben nur relativ kleine Verbände von Langobarden ebenso wie Erulern in Südmähren und an der Donau die ethnische Kontinuität verkörpert, während der Hauptteil der ansässigen wie der zugewanderten Bevölkerung sich zunächst weder zum einen noch zum anderen Volk bekannte. Jedenfalls ist in Noricum und Pannonien mit einer neuen Ethnogenese der Langobarden zu rechnen. ${ }^{32}$

Reichsbildung und Integration in Italien bewirkten weitere grundlegende Wandlungen, die teils ganz allmählich und von zeitgenössischen Autoren weitgehend unbemerkt vor sich gingen. Für die aktuelle archäologische Debatte ist die langobardische Invasion in Italien ein wichtiges Exempel für die Möglichkeit ethnischer Deutung einer Fundgruppe zumindest unter bestimmten Voraussetzungen. In Pannonien endet eine bestimmte archäologische Kultur etwa zum selben Zeitpunkt, als in Italien ähnliche Gräberfelder einsetzen. Dieser eindeutige archäologische Befund kann nun (ohne zuvor ,vermischte Argumentation' gebraucht zu haben) mit dem aus den Schriftquellen datierbaren langobardischen Zug nach Italien von 568/69 in Verbindung gebracht werden. Es ist naheliegend, ein ,langobardisches Kulturmodell ' in Italien anzunehmen. ${ }^{33}$

Die Definition dieses langobardischen Kulturmodells beruht vor allem auf dem Totenbrauchtum, und dabei in erster Linie auf der Waffenbeigabe, die freilich nur in relativ wenigen italienischen Gräberfeldern (z. B. in Nocera Umbra) tatsächlich signifikant belegt ist. ${ }^{34}$ Nach dieser Definition wären die Langobarden in vollem Sinn also (selbst wenn man den schlechten Forschungs- und Publikationsstand der Langobardenarchäologie in Italien in Betracht zieht) eine relativ kleine Kerngruppe. Größere Verbreitung

40 (ed. Allan A. Lund, Wissenschaftliche Kommentare zu griechischen und lateinischen Schriftstellern, Heidelberg 1988) 100 (Langobardos paucitas nobilitat); Tacitus, Annalen 2, 45f.; 11,17 (ed. Heinrich Heubner, Bibliotheca scriptorum Graecorum et Romanorum Teubneriana, Stuttgart 1983) 70, 222; Ptolemaios, Geographia 2, 11, 9 (ed. Carl Friedrich August Nobbe, Leipzig 1843-1845, Nachdruck: Hildesheim 1966) 117.

${ }_{30}$ Walter Pohl, Überlegungen zur ethnischen Kontinuität zwischen Spätantike und Frühmittelalter, in: Kontinuität und Diskontinuität in der frühgeschichtlichen Entwicklung im Mitteldonauraum, ed. Jaroslav Tejral, im Druck.

${ }^{31}$ Jaroslav Tejral, Archäologischer Beitrag zur Kenntnis der völkerwanderungszeitlichen Ethnostrukturen nördlich der Donau, in: Typen der Ethnogenese unter besonderer Berücksichtigung der Bayern 2, ed. Herwig Friesinger/Falko Daim (Denkschriften der Österreichischen Akademie der Wissenschaften 204, Wien 1990) 9-88; Frauke Stein, „Da erhoben sich die Langobarden aus ihren Sitzen und wohnten etliche Jahre in Rugiland“. Zu den Grenzen von Siedlungsräumen nach schriftlichen und archäologischen Quellen, in: Grenzen erkennen - Begrenzungen überwinden. Festschrift Reinhard Schneider, ed. Wolfgang Haubrichs/ Karl-Ulrich Jäschke/Michael Oberweis (Sigmaringen 1999) 35-101; darauf antwortet wiederum der Beitrag von Jaroslav Tejral, in diesem Band.

32 Jarnut, Die langobardische Ethnogenese.

${ }_{33}$ Volker Bierbrauer, Die Landnahme der Langobarden in Italien aus archäologischer Sicht, Ausgewählte Probleme europäischer Landnahmen des Früh- und Hochmittelalters: methodische Grundlagendiskussion im Grenzbereich zwischen Archäologie und Geschichte 1, ed. Michael Müller-Wille/Reinhard Schneider (Vorträge und Forschungen 41, Sigmaringen 1993) 103-172. Siehe auch den Beitrag von Volker Bierbrauer in diesem Band.

${ }_{34}$ Volker Bierbrauer, Frühe langobardische Siedlung in Italien: Das Problem der Gräber- und Siedlungsarchäologie, in: I Longobardi dei ducati di Spoleto e Benevento (Atti del XVI congresso di studi sull'alto medioevo, Spoleto 2003, im Druck); vgl. Cornelia Rupp, Langobardische Gräberfelder in Umbrien, ebd. 
hat bis in die erste Hälfte des 7. Jahrhunderts die gestempelte ,langobardische' Keramik, wie sie etwa in der Umgebung von S. Giulia in Brescia in großer Zahl gefunden wurde. ${ }^{35}$ Die Versuchung ist groß, zu diesen recht deutlichen Befunden andere, wesentlich weniger aussagekräftige Elemente hinzuzufügen, um auch in zweifelhafteren Fällen eine langobardische Zuordnung begründen zu können. Doch das methodische Problem besteht darin, dass viele ,langobardenzeitliche 'Befunde in Italien eben nicht eindeutig ,langobardisch“ sind; auch die ,romanische“ Identität lässt sich, bedingt gerade durch die Kargheit der Grabfunde, weniger deutlich fassen. Der archäologische Befund sollte also nicht als Beweis für eine geschlossene langobardische Identität der Wanderzeit und der ersten Jahrzehnte in Italien überbewertet werden. Dahinter verbirgt sich eine abgestufte Vielfalt von lokalen und regionalen Befunden, die keineswegs eine schematische Gegenüberstellung von ,Langobarden“ und ,Romanen“ nahelegen; selbst in der „Landnahmegeneration“ im letzten Drittel des 6. Jahrhundert hat sich das Bild gegenüber den pannonischen Langobarden bereits differenziert. ${ }^{36}$

Diese Überlegungen stellen die Möglichkeit, archäologische Spuren der Langobarden in Italien zu finden, nicht prinzipiell in Frage; dass in den Waffengräbern von Nocera Umbra zumindest überwiegend Langobarden bestattet sind, scheint naheliegend. Doch hat die Archäologie keinen direkten Zugriff auf langobardische Identität. Das ,langobardische Kulturmodell' gibt sicherlich einen guten Anhaltspunkt für Grabsitten und kulturelle Muster, die unter den langobardischen Führungsgruppen verbreitet waren. Das entspricht ungefähr einer Definition ethnischer Identität, die auch in frühmittelalterlichen Schriftquellen verbreitet war und die das Heer und die politisch Mitwirkungsberechtigten umfasste. ${ }^{37}$ Das war ungefähr jene Gruppe, auf deren Konsens sich Rothari bei der Promulgation seines Edikts beruft: primatos iudices cunctosque felicissimus exercitum nostrum. ${ }^{38}$ Doch archäologisch kann nicht ausgeschlossen werden, dass Mitglieder dieser Gruppe sich bereits bald nach 568 ohne Beigaben bestatten ließen und daher nicht dem langobardischen Kulturmodell folgten. Beim derzeitigen Forschungsstand ist das nicht einmal unwahrscheinlich, bedenkt man, wie selten Gräberfelder vom Typ von Nocera Umbra gerade im langobardischen Kernraum in der Lombardei zu sein scheinen. Langobarden im weiteren Sinn, nämlich unter Einschluss derjenigen, die den Aufstieg in die herrschende Kriegerschicht nicht geschafft hatten, sind archäologisch umso schwerer zu definieren und z. B. von anderen barbarischen Bevölkerungsgruppen zu unterscheiden. Wie würde man die ancilla gentile des Edikts archäologisch von der ancilla Romana unterscheiden ${ }^{39}$ Die Archäologie argumentiert, aus historischer Sicht, dort am überzeugendsten, wo die Vielfalt des Materials und der Befunde nicht einfachen Gegensatzpaaren (langobardisch/germanisch-romanisch) untergeordnet wird.

Geht man nach den Kriterien, die Isidor und andere frühmittelalterliche Gelehrte für ethnische Zugehörigkeit formuliert haben: arma, habitus, mores, lingua, leges, und ähnliches, ${ }^{40}$ so hatte sich all das, teils bereits mehrfach, gewandelt, als Paulus Diaconus

${ }_{35}$ Vgl. Gian Pietro Brogiolo, Brescia altomedievale. Urbanistica ed edilizia dal IV al IX secolo (Mantova 1993).

${ }_{36}$ Siehe den Beitrag von Irene Barbiera in diesem Band; sowie ihre Dissertation zu diesem Thema, die 2003 an der Central European University in Budapest angenommen wurde: dies., Migration and Identity during the Lombard Invasions.

${ }_{37}$ Vgl. Pohl, Zur Bedeutung ethnischer Unterscheidungen.

38 Edictus Rothari 386 (ed. Friedrich Bluhme, MGH LL 4, Hannover 1868) $89 \mathrm{f}$.

${ }_{39}$ Edictus Rothari 194, ed. Bluhme 47. Gasparri, Prima delle nazioni 149-151, argumentiert im übrigen eindrucksvoll, dass die ancilla Romana nicht unbedingt selbst römischer Herkunft sein muß, sondern darunter auch die ancilla eines römischen Herrn zu verstehen sein könnte, parallel zur ancilla Dei in Liutprand 95 , die ebenfalls der ancilla gentile gegenübergestellt wird.

40 Pohl, Telling the difference $17 \mathrm{f}$. 
vor 800 seine Langobardengeschichte schrieb. Er selbst beobachtete, dass sich die Tracht der Langobarden seiner Zeit gegenüber den Wandmalereien im Palast der Theodelinde in Monza völlig geändert hatte. ${ }^{41}$ Über die Veränderung von Habitus und Sitte kann die Archäologie wesentliche Aussagen machen; schon der Grabbrauch durchlief mehrfach grundlegenden Wandel: in der Spätantike die Durchsetzung der Körperbestattung, bei den pannonischen Langobarden die Verbreitung der Reihengräbersitte, ${ }^{42}$ und schließlich im Lauf des 7. Jahrhunderts das Verschwinden der Grabbeigaben, und zwar bei Frauen meist früher als bei Männern. ${ }^{43}$ In wenigen Generationen verschob sich der Grabbrauch „dai corredi funerari alle donazioni post mortem “. ${ }^{44}$ Bei der langobardischen Tracht kommt das methodische Problem dazu, dass viele Trachtbestandteile ausschließlich aus den Gräbern der Langobarden (oft auch anderer Völker) erhalten sind; es ist aber schwer nachzuweisen, dass nicht auch Romanen ähnliche Tracht trugen, aber eben nicht mit ins Grab legen. In den Werkstätten der Cripta Balbi in Rom wurden Reste ,langobardischer" Fibeln gefunden, die derartige Fragen aufwerfen: oder handelte es sich um Exportgüter ${ }^{45}$ In Rom belegt auch ein Fresko in Sta. Maria Antiqua vom Beginn des 8. Jahrhunderts die Verwendung mehrteiliger Gürtelgarnituren, wie sie sonst aus bayerischen und awarischen Gräbern bekannt sind. ${ }^{46}$ Ohne Zweifel waren die herrschenden Langobarden auf ethnische und soziale Distinktion bedacht, aber welche Zeichen und Symbole dazu tatsächlich dienten, ist schwer nachzuweisen. Der langobardischen Identität tat Wechsel von Tracht und Sitten jedenfalls keinen Abbruch, ebensowenig wie die Übernahme des katholischen Bekenntnisses und die Aufgabe der hömöischen Lehre und des Heidentums.

Was Paulus, obwohl einer der führenden Grammatiker seiner Zeit, nirgends erwähnt hat, ist das Verschwinden der langobardischen Sprache. Meist führt er langobardische Begriffe mit Formeln ein wie lingua propria dicunt, „sie sagen in ihrer Sprache“, oder sogar sermone barbarico appellatur, „es heißt in barbarischer Rede“. ${ }^{77}$ Die einmal gebrauchte Formulierung quem vulgo scaffardum dicimus findet sich als Zusatz in einer einzigen Handschrift des 14. Jahrhunderts. ${ }^{48}$ Diskutierbar ist, ob die langobardische Sprache bereits im 8. Jahrhundert außer Gebrauch geriet oder erst im 9. Jahrhundert; viel länger hielt sich nur die Tradition der langobardischen Personennamen, auch wenn sie nicht unbedingt die ethnische Zugehörigkeit anzeigen mussten. ${ }^{49} \mathrm{Im}$ 10./11. Jahr-

${ }^{41}$ Paulus Diaconus, Historia Langobardorum 4, 22, ed. Waitz 124. Vgl. Pohl, Telling the difference $43 \mathrm{f}$.

${ }^{42}$ Joachim Werner, Die Langobarden in Pannonien (München 1962).

${ }^{43}$ Siehe den Beitrag von Volker Bierbrauer, in diesem Band.

${ }^{44}$ Cristina la Rocca, Segni di distinzione. Dai corredi funerari alle donazioni 'post mortem' nel regno longobardo, in: L'Italia centro-settentrionale in età longobarda, ed. Lidia Paroli (Florenz 1997) 31-54, hier 54.

${ }_{45}$ Marco Ricci/Federica Luccerini, Oggetti di abbigliamento e ornamento, in: Roma dall'antichità al medioevo. Archeologia e storia nel Museo nazionale romano Crypta Balbi, ed. Maria Stella Arena u. a. (Rom 2001) 351-387, bes. 380-385.

${ }^{46}$ Falko Daim, ,Byzantinische Gürtelgarnituren des 8. Jahrhunderts, in: Die Awaren am Rand der byzantinischen Welt, Studien zu Diplomatie, Handel und Technologietransfer im Frühmittelalter, ed. Falko Daim (Monographien zur Frühgeschichte und Mittelalterarchäologie 7, Innsbruck 2000) 77-204.

${ }_{47}$ Z. B. Paulus Diaconus, Historia Langobardorum 1, 9, ed. Waitz 59; ebd. 1, 15, ed. Waitz 55; ebd. 1, 20, ed. Waitz 57 und 59; ebd. 2, 9, ed. Waitz 77. Siehe Nicholas Everett, Literacy in Lombard Italy, c. 568-774 (Cambridge 2003) 110-112.

${ }_{48}$ Paulus Diaconus, Historia Langobardorum 5, 2, ed. Waitz 143 (Handschrift L 2). Siehe Nicholas Everett, Literacy in Lombard Italy 110-112.

${ }_{49}$ Nicoletta Frankovich Onesti, Vestigia longobarde in Italia (568-774). Lessico e antroponimia (Rom 1999) 48, für das Verschwinden des Langobardischen bereits im 8. Jahrhundert oder früher; anders Wolfgang Haubrichs, in diesem Band. Zum Problem der Identitätswirksamkeit von Personennamen siehe allgemein Jörg Jarnut, Selbstverständnis von Personen und Personengruppen im Lichte frühmittelalterlicher Personennamen, in: ders., Herrschaft und Ethnogenese im Frühmittelalter. Gesammelte Aufsätze, ed. Matthias Be- 
hundert benützte man in Süditalien jedenfalls Glossare für die langobardischen Rechtswörter, deren drastische Verschreibungen ihre Fremdartigkeit belegen. ${ }^{50}$ Das langobardische Recht hatte sich bei aller rhetorischen Beschwörung seiner Ehrwürdigkeit durch die lateinische Kodifizierung und ständige Weiterentwicklung im 7. und 8. Jahrhundert ebenfalls beträchtlich geändert. All das könnte noch im einzelnen ausgeführt und differenziert werden; es führt zur Frage: was machte eigentlich die langobardische Identität aus?

Darauf gibt es zunächst eine naheliegende Antwort: der Volksname. Bei den Langobarden in Italien gibt es keinen Zweifel darüber, dass er durchgehend Fremd- wie Selbstbezeichnung war, auch wenn die Selbstbenennung als Langobarden erst im 7. Jahrhundert zweifelsfrei nachweisbar ist. Außergewöhnlich ist bei den Langobarden zudem die enge Verbindung zwischen Namen und Herkunftsmythos, der ja vor allem die Namengebung erklärt. ${ }^{51}$ Bei näherer Betrachtung gibt es freilich einen Widerspruch zwischen dem Mythos und der üblichen etymologisch fundierten Erklärung, also zwischen Origo gentis und Etymologia sive Origo im Sinn Isidors. In der Herkunftssage sind es die Frauen, deren falsche Bärte Wodan zur Namengebung veranlassen; nach Isidor die prolixa barba et numquam tonsa der Männer, eine Erklärung, die sich durchgesetzt hat (auch bei Paulus Diaconus). ${ }^{52}$ Der Erfolg der Etymologie liegt auch daran, dass sie im Lateinischen ebenso evident bleibt: qui sunt isti longibarbae, fragt Wodan im lateinischen Text der Origo gentis Langobardorum. Als pragmatisches Erkennungszeichen sind lange Bärte freilich ebenso problematisch wie lange Haare, weil sie als Symbol polyvalent sind; es genügt, an die byzantinischen Münzen des 7. Jahrhunderts zu erinnern, auf denen die Kaiser sich durch lange Bärte auszeichnen. ${ }^{53}$ Dennoch behielt die Barttracht ihre symbolische Bedeutung. Ein knapper Auszug des 13. Jahrhunderts aus der Langobardengeschichte des Paulus fügte hinzu: Verum quia Lombardi dimiserunt barbam longam et capillos more feminarum, ipsam quoque ferro incidere facientes, dicti sunt solummodo Lombardi, cum ante dictifuissent Longobardi. ${ }^{54}$ Indem sie den Bart abrasierten, wurden aus den Langobarden die Lombarden; die Ambivalenz der weiblichen Origo der langen Bärte (more feminarum, heißt es hier über die langen Haare) konnte nun zur Absetzung von der barbarischen Herkunft der Langobarden genützt werden. Der Versuch, die lombardische von der langobardischen Identität abzuheben, gipfelte in der Geschichte, nach der die Lombarden die langobardischen Invasoren unter Gambara aus dem Land geworfen hätten. ${ }^{55}$

cher (Münster 2002) 355-373; für die Langobarden Maria Giovanna Arcamone, Die langobardischen Personennamen in Italien: nomen und gens aus der Sicht der linguistischen Analyse, in: Nomen et gens. Zur historischen Aussagekraft frühmittelalterlicher Personennamen, ed. Dieter Geuenich/Wolfgang Haubrichs/Jörg Jarnut (RGA Erg. Bd. 16, Berlin/New York 1997) 157-175; sowie Wolfgang Haubrichs, in diesem Band. Belege für lateinische Namen von Langobarden und umgekehrt bei Brigitte Pohl-Resl, Ethnische Bezeichnungen und Rechtsbekenntnisse in langobardischen Urkunden, in: Ethnogenese und Überlieferung: Angewandte Methoden der Frühmittelalterforschung, ed. Karl Brunner/Brigitte Merta (Veröffentlichungen des Instituts für Österreichische Geschichtsforschung 31, Wien 1994) 163-171.

${ }_{50}$ Federico Albano Leoni, Tre glossari longobardo-latini (Napoli 1981); Pohl, Werkstätte der Erinnerung 199-208.

${ }^{51}$ Dazu und zum folgenden Pohl, Origo gentis (Langobarden). Vgl. auch den Beitrag von Robert Nedoma in diesem Band.

${ }_{52}$ Isidor, Etymologiae 9, 2, 95 (ed. Wallace M. Lindsay, 2 Bde., Oxford $\left.{ }^{2} 1987\right)$; Paulus Diaconus, Historia Langobardorum 1, 9, ed. Waitz 52: ab intactae ferro barbae longitundinis. Vgl. künftig Walter Pohl, Gender and ethnicity in the early middle ages, in: Gender in the Early Medieval World. East and West, 300-900, ed. Leslie Brubaker/Julia Smith (Cambridge 2004) 23-43.

${ }_{53}$ Pohl, Telling the difference 56-61.

${ }_{54}$ Istoria Langobardorum aus Cod. Paris BN 4931 (ed. Georg Waitz, MGH rer. Lang., Hannover 1878) $196 \mathrm{f}$., hier 196.

${ }_{55}$ Jörg Busch, Die Lombarden und die Langobarden, in: Frühmittelalterliche Studien 29 (1995) 289-311. 
Das Beispiel des Langobardennamens ist gerade deshalb lehrreich, weil es erlaubt, ein Stück weit in die Vorstellungen vorzudringen, die sich damit verknüpfen. Dort aber beginnen sofort Ambivalenzen und Widersprüche. Man stößt auf weibliche Bärte und auf Erkennungszeichen, die kein eindeutiges Erkennen ermöglichen können. Und dennoch gab es die Langobarden. Viele von ihnen trugen sicherlich lange Bärte. Nur, und das ist ein wesentlicher Punkt, um den es hier geht: Unsere Ordnungsvorstellungen greifen noch weniger als die unsystematischeren Kategorien jener frühmittelalterlichen Autoren, die selbstverständlich den Begriff Langobarden gebrauchten. Was den Langobarden außer dem Namen gemeinsam war, ließ sich zwar als Anspruch formulieren: lange Bärte, Freiheit, Kriegertum, gemeinsame Herkunft und Tradition. Doch hinter diesem vorherrschenden Modell langobardischer Identität lag eine reale Vielfalt der Lebensformen, die für Freiheit und Unfreiheit, Krieger und Nichtkrieger, Bartträger und Rasierte und Menschen verschiedener Herkunft Platz bot. Durch Integration der Romanen in den Langobardenbegriff entstand schließlich die Lombardei als territoriale Identität im Gegensatz zur Romagna.

Diese Spannung zwischen ständigem pauschalem und ideologischen Gebrauch des Langobardennamens und seiner eingeschränkten pragmatischen Bedeutung wird beim langobardischen Recht deutlich. Wozu diente der Langobardenname in den Leges? Der Befund ist deutlich. Erstens erscheint er in den Prologen im Königstitel; zweitens umschreibt er in den Prologen, aber auch in einigen Gesetzen die fideles Langobardi, an die sich die königliche Gesetzgebung richtet und auf deren Konsens sie abzielt; drittens dient er zur Bezeichnung der lex (manchmal auch consuetudo) Langobardorum (meist formelhaft, nur bei Liutprand einmal im Gegensatz zur lex Romanorum); ${ }^{56}$ und viertens führt Liutprand, im 8. Jahrhundert, die einleitende Formel Si quis Langobardus ein, gefolgt von einem Tatbestand und der Sanktion. Nur ganz selten wird der Volksname tatsächlich zur Distinktion gebraucht; das ist im Gesetz Liutprands der Fall, das die Ehe zwischen einem Römer und einer mulier Langobarda regelt, wodurch die Frau romana effecta est. ${ }^{57}$ Rothari hatte noch der ancilla Romana - bekanntlich die einzige Erwähnung von Römern in seinem Edikt - die höherwertige ancilla gentile gegenübergestellt. ${ }^{58}$

Solche Fälle der Abgrenzung und des zumindest möglichen Konflikts sind es auch, die mehr über Umrisse und Bedeutung der langobardischen Identität erkennen lassen. Wenn Liutprand die Formel Si quis Langobardus einführt, muss gefragt werden, warum nötig wurde, das zu betonen. Viele Handschriften der Leges lassen Langobardus an dieser Stelle freilich weg, und auch das mag seinen Grund haben. Aber selbst im Konfliktfall wurde nicht unbedingt die langobardische Identität gegenüber der römischen innerhalb des Reiches hervorgehoben. Als Aistulf im Krieg mit Byzanz eine Bestimmung erließ, nach der jeder Handel mit Römern (in diesem Fall Untertanen des Exarchates) untersagt war, sah er durchaus drastische Sanktionen vor. Jeder Arimanne, der dagegen verstieß, sollte seine Güter verlieren und öffentlich geschoren werden, wobei man ausrief: „Sic patiatur, qui contra voluntatem regis cum Romano homine negotium fecerit, quando lites habemus ". ${ }^{59}$ Dass es um Langobarden ging, wird nicht gesagt; wesentlich ist hier die soziale Identität (iudex, Arimanne oder anderer homo), nicht die ethnische.

${ }_{56}$ Liutprandi Leges 91, ed. Bluhme $144 \mathrm{f}$.

${ }_{57}$ Liutprandi Leges 127, ed. Bluhme 160: Si quis Romanus homo mulierem langobardam tolerit.

${ }_{58}$ Edictus Rothari 194, ed. Bluhme 47. Si quis cum ancilla gentile fornicatus fuerit, conponat domino eius solidos viginti; si cum Romana ancilla, conponat sold. duodicem.

${ }_{59}$ Ahistulfi Leges 4 (ed. Friedrich Bluhme, MGH LL 4, Hannover 1868) 196f. Siehe Walter Pohl, Frontiers in Lombard Italy: the laws of Ratchis and Aistulf, in: The Transformation of Frontiers - from Late Antiquity to the Carolingians, ed. Walter Pohl/Ian Wood/Helmut Reimitz (The Transformation of the Roman World 10, Leiden 2000) 117-142. 
Bei den langobardischen Privaturkunden, die näher an die Rechtswirklichkeit heranführen, kommt der Langobardenname so gut wie gar nicht vor. Das mag seinen Grund darin haben, dass bei Rechtsgeschäften unter Langobarden die ethnische Identität (und das Rechtsbekenntnis) nicht betont werden mussten. Erst 768, also kurz vor Ende des selbständigen Königreiches, erscheint erstmals die später so häufige Formel secundum legem Langobardorum. ${ }^{60}$ Doch hat sich das seit dem 9. Jahrhundert völlig geändert; langobardisches Rechtsbekenntnis und Verweis auf die Leges erreichten lange nach dem Ende des Langobardenreiches, im 11. Jahrhundert, ihren Höhepunkt. In den Königsurkunden der Langobardenzeit wird auch nicht der Königstitel der Leges, rex gentis Langobardorum, sondern der römisch-gotische Titel flavius rex gebraucht. ${ }^{61}$ Die Selbstdarstellung des langobardischen Königtums zielte keineswegs darauf ab, den langobardischen Charakter seiner Herrschaft immer in den Vordergrund zu rücken. Das Gedicht eines Geistlichen über die Beendigung des Drei-Kapitel-Schismas um 700 betont stolz, dass die Langobardenkönige aus vornehmer Familie in finibus Europe stammten, und hebt hervor, dass die Langobardenkönige der zweiten Hälfte des 7. Jahrhunderts „fromm und katholisch“ gewesen seien und in jeder Weise die Kirche gefördert hätten. ${ }^{62}$ Um ethnische Distinktion geht es hier gerade nicht. Im Übrigen entsteht der Eindruck, dass die rhetorische Bedeutung der langobardischen Identität ihre pragmatische bis ins 8. Jahrhundert überstieg. Als Kategorie abgrenzbar wurde der Langobardenbegriff erst dann, als er nach der karolingischen Machtübernahme seine ideologische Funktion einbüßte. Die ethnische Identität in den Regna war eher einschließend als ausschließend. Das daran geknüpfte politische Ziel war Integration und Loyalität aller wesentlichen Kräfte, nicht die Ausgrenzung der Nicht-Langobarden.

Der Zusammenhalt der Gens war nicht zuletzt die Leistung des regnum Langobardorum. Das Interregnum von 574 bis 584 zeigt, dass das nicht selbstverständlich war; noch in den 570er Jahren rechnete Papst Pelagius II. damit, die Langobarden würden sich "wie der Rauch" verziehen. ${ }^{63}$ Von Königin Theodelinde ist bezeugt, wie sehr sie sich um langobardische Geschichte und Identität bemühte. ${ }^{64}$ Texte (darunter die wenigen, die uns erhalten sind) können nicht nur das Zugehörigkeitsbewusstsein bestimmter Bevölkerungsgruppen abbilden, sie tragen unter Umständen selbst dazu bei, es zu formen. Viele frühmittelalterliche Geschichtsschreiber waren ja keineswegs Stubengelehrte, sondern hohe Amtsträger oder Berater der Herrschenden (wie Secundus bei Theodelinde). Anthony D. Smith hat vor kurzem die jüngere Debatte über Ethnizität durch die

${ }_{60}$ Dazu und zum folgenden: Brigitte Pohl-Resl, Legal practice and ethnic identity in Lombard Italy, in: Strategies of Distinction. The Construction of Ethnic Communities, 300-800, ed. Walter Pohl/Helmut Reimitz (The Transformation of the Roman World 2, Leiden/New York/Köln 1998) 205-220.

${ }^{61}$ Herwig Wolfram, Intitulatio I. Lateinische Königs- und Fürstentitel bis zum Ende des 8. Jahrhunderts (MIÖG Erg. Bd. 21, Köln/Wien 1967) 90-107.

${ }_{62}$ Rhythmus de synodo Ticinensi (ed. Ludwig Bethmann, MGH SS rer. Langob., Hannover 1878) $190 \mathrm{f}$. oder (ed. Karl Strecker, MGH Poetae 4, 2, München ${ }^{2} 1923$ [Neudruck München 1978]) 728-73: Sublimes ortus in finibus Europe/Langibardorum regali prosapia. Zum Rhythmus siehe den Beitrag von Kurt Smolak in diesem Band. Der (moderne) Titel ist insofern irreführend, als von einer Synode darin gar nicht die Rede ist; siehe künftig Walter Pohl, Heresy in Secundus and Paul the Deacon, in: The Crisis of the Oikoumene: The Three Chapters and the Failed Quest for Unity in the Sixth-Century Mediterranean, ed. Celia Chazelle/ Catherine Cubitt (im Druck).

${ }_{63}$ Epistulae Aevi Merowingici Collectae 9 (ed. Wilhelm Gundlach, MGH EE 3, Merovingici et Karolini aevi 1, Berlin 1892) 434-468, hier 449. Vgl. Walter Pohl, The Empire and the Lombards: treaties and negotiations in the sixth century, in: Kingdoms of the Empire. The Integration of Barbarians in Late Antiquity, ed. Walter Pohl (The Transformation of the Roman World 1, Leiden/New York/Köln 1997) 75-134, bes. $101 \mathrm{f}$.

${ }_{64}$ Walter Pohl, Memory, identity and power in Lombard Italy, in: The Uses of the Past in the Early Middle Ages, ed. Yitzhak Hen/Matthew Innes (Cambridge 2000) 9-28; vgl. Ross Balzaretti, Theodelinda, 'Most glorious Queen': gender and power in Lombard Italy, in: Medieval History Journal 2, 2 (1999) 183-207. 
Auseinandersetzung zweier Positionen charakterisiert. ${ }^{65}$ Die eine (die er selbst vertritt) nennt er ethnosymbolic; sie sieht in der langfristigen kulturellen Aneignung und Umformung der Vergangenheit in der Form identitätsstiftender Mythen den Kern nationaler Identität. Die andere bezeichnet er als social constructionism, die von der bewussten Manipulation ethnischer Identitäten durch invented traditions und kulturelle Fiktionen ausgeht. Im Frühmittelalter ist dieser Gegensatz schwer nachzuvollziehen: Aneignung und Neuprägung der Vergangenheit, schöpferisches Traditionsbewusstsein und bewusste Traditionsschöpfung greifen, für uns oft ununterscheidbar, ineinander.

Das lässt sich auch an der langobardischen Geschichte zeigen, die nach 800 weiter gebraucht, verändert, immer neu erzählt und gelesen wurde. Langobardische Identität blieb in Nord- und Süditalien, wenn auch mehrfach gebrochen, gemeinschaftsstiftend. Seit der Karolingerzeit können wir den Umgang mit langobardischer Identität leichter fassen, weil nun die handschriftliche Überlieferung stark anwächst. Bisher hat man dieses reiche Material kaum benützt und sich bei der Suche nach identitätsrelevanten Texten an edierte Quellen gehalten. Die einigermaßen datier- und zuordenbaren Handschriften können systematisch in die Untersuchung einbezogen werden, wie ich es in meiner Studie über Montecassino als „Werkstätte der Erinnerung“ versucht habe oder wie es Helmut Reimitz bei der fränkischen Historiographie unternimmt. ${ }^{66}$ In Süditalien war, bis zur Entmachtung durch die Normannen, die langobardische Identität (und langobardische Personennamen) wichtig für die privilegierte Stellung der längst romanisierten Führungsgruppen und für den stets bedrohten Zusammenhalt der konfliktgeschüttelten Fürstentümer. Ob man die Langobarden damals noch an ihren Bärten erkennen konnte, darf bezweifelt werden. Doch im Spannungsfeld von Text und Identität erinnerte sich wohl nicht nur Rampho an eine Geschichte, die sich in ferner Vorzeit zugetragen hatte und die erklärte, wie aus den mythischen Winnilern Langobarden geworden waren.

\footnotetext{
${ }_{65}$ Anthony D. Smith, The Nation in History. Historiographic Debates about Ethnicity and Nationalism (Hanover, NH 2000).

${ }_{66}$ Pohl, Werkstätte der Erinnerung; Helmut Reimitz, Ein fränkisches Geschichtsbuch aus Saint für Österreichische Geschichtsforschung, ed. Christoph Egger/Herwig Weigl (MIÖG Erg. Bd. 35, Wien 1999) 34-90.
} 